\title{
Detecção de Estímulos Radiais e Espaciais em Adultos e Idosos ${ }^{1}$
}

\author{
Natanael Antonio dos Santos 2 \\ Adriana Bastos Oliveira \\ Universidade Federal da Paraíba \\ Renata Maria Toscano Barreto Lyra Nogueira \\ Universidade de São Paulo \\ Melyssa Kellyane Cavalcanti \\ Universidade Federal da Paraíba \\ Maria Lúcia de Bustamante Simas \\ Universidade Federal de Pernambuco
}

\begin{abstract}
RESUMO - O objetivo deste estudo foi comparar curvas de sensibilidade ao contraste para estímulos radiais (FSCr) e grades senoidais (FSC) de 0,25, 0,5, 1 e $2 \mathrm{cpg}$ em adultos e idosos. Mensuramos limiares de contraste para seis adultos jovens e seis idosos utilizando o método psicofísico da escolha forçada. Todos estavam livres de doenças oculares e tinham acuidade visual normal. Os idosos apresentaram prejuízos na FSC e FSCr se comparados aos adultos jovens. A sensibilidade dos adultos e idosos foi maior para grades senoidais (FSC) do que para estímulos radiais (FSCr). Esses resultados sugerem que esses estímulos podem ser processados por áreas visuais distintas.
\end{abstract}

Palavras-chave: percepção visual; sensibilidade ao contraste; envelhecimento humano; freqüência radial e espacial; método da escolha forçada.

\section{Detection of Spatial and Radial Stimuli in Young and Older Adults}

\begin{abstract}
The aim of this work was to compare contrast sensitivity curves for radial (rCSF) and sine-wave gratings (CSF) stimuli of $0.25,0.5,1$ and $2 \mathrm{cpd}$ in young and older adults. We measured the contrast thresholds for six young and six older adults using the psychophysical forced-choice staircase method. All were free of ocular diseases and had normal visual acuity. The older adults presented a loss in rCSF and FSC if compared to the young adults. The contrast sensitivity for young and older adults was higher for spatial frequencies (CSF) than radial frequencies (rCSF). These results suggest that these stimuli can be processed by distinct visual areas.
\end{abstract}

Key words: visual perception; contrast sensitivity; human aging; radial and spatial frequency; forced-choice method.

Uma das principais perspectivas teóricas utilizadas para investigar como o sistema visual humano $(\mathrm{SVH})$ detecta e reconhece objetos (forma ou contraste) é denominada modelo de canais múltiplos. Essa abordagem proposta originalmente por Campbell e Robson (1968) assume que a retina inicialmente decompõe (ou analisa) os objetos em componentes elementares (p.ex., freqüências espaciais) que são processados por vias que partem da retina passando pelo núcleo geniculado lateral até alcançar o córtex visual no qual os objetos são recompostos (ou sintetizados). Nessa perspectiva, o processamento ou a percepção de uma cena qualquer ocorre porque o SVH executa uma análise desse padrão complexo em seus componentes mais elementares que são posteriormente sintetizados e identificados pelo

1 Trabalho apresentado na XX Reunião Anual da Federação de Sociedades de Biologia Experimental (FeSBE). Pesquisa financiada pelo CNPq através dos processos 307182/2003-7 e 475064/2004-6.

2 Endereço: Departamento de Psicologia, CCHLA - UFPB, Campus I, Cidade Universitária, João Pessoa, PB, Brasil 58051-900. E-mail: natanael.santos@pesquisador.cnpq.br córtex visual (Blakemore \& Campbell, 1969a, 1969b; Campbell \& Robson, 1968; De Valois \& De Valois, 1988; Graham, 1972; Graham \& Nachmias, 1971; Sachs, Nachmias \& Robson, 1971).

Atualmente, estudos psicofísicos em humanos e estudos neurofisiológicos em macacos fornecem evidências empíricas para o modelo de canais múltiplos ou mecanismos sintonizados para banda estreita de freqüências espaciais (Dakin \& Hess, 1997; Menees, 1998; Olds \& Engel, 1998; Santos \& Simas, 2002; Santos, Simas \& Nogueira, 2004; Simas \& Santos, 2002a, 2002b; Wilson, Levi, Maffei, Rovamo \& De Valois, 1990). Esses estudos pressupõem que a função de sensibilidade ao contraste (FSC) representa o envelope da sensibilidade para a série total de canais, cada um sensível a uma região do espectro (Braddick, Campbell \& Atkinson, 1978; De Valois \& De Valois, 1988). Um canal, do ponto de vista psicofísico, é, por exemplo, um filtro com estrutura independente no sistema que lida com uma faixa estreita de freqüência espacial. Do ponto de vista fisiológico, um canal significa uma população neuronal sintonizada para uma banda-estreita de freqüência espacial (Santos \& Simas, 2001, 2002). 


\section{Função de sensibilidade ao contraste (FSC)}

A sensibilidade ao contraste é definida como a recíproca da quantidade mínima de contraste necessária para detectar um objeto qualquer (p. ex., uma grade) de uma determinada frequiência espacial (Cornsweet, 1970). Assim, na mensuração da FSC, o limiar de contraste para cada freqüência espacial é ajustado com um procedimento comportamental ou psicofísico até que o SVH possa detectar um padrão com uma dada freqüência. Nesses termos, as variáveis experimentais principais são frequiência espacial (variável independente) e contraste (variável dependente). Freqüência espacial é definida como o número de ciclos (ou listras claras e escuras) por grau de ângulo visual (cpg). Enquanto contraste é a diferença entre a luminância máxima (listra clara) e mínima (listra escura) da grade (ver Figura 1) dividido pela soma de ambas.

Uma característica importante da FSC advém do fato que a sua forma muda em função 1) das condições visualizadas (Benedek, Benedek, Kéri \& Janáky, 2003; De Valois. \& De Valois, 1988; Geogeson \& Sullivan, 1975), 2) da fase de desenvolvimento (Candy, Crowell \& Banks, 1998; Van Sluyters, Atkinson, Held, Hoffman \& Shatz, 1990) e 3) de desordens neuropatológicas (Akutsu \& Legge, 1995; Bour \& Apkarian, 1996; Elliott \& Situ, 1998; O’ Donnell \& cols., 2002; Slaghuis, 1998), o que permite fortes inferências sobre o funcionamento de mecanismos fisiológicos básicos relacionados ao processamento visual de objetos e/ou de detalhes espaciais em situações diversas. Por essas e outras características, a FSC é uma medida clássica na avaliação teórica e clínica do SVH (John, 1997; Wilson \& cols., 1990).

\section{Detecção de estímulos radiais circularmente simétricos}

Em 1960 Kelly sugeriu a utilização de estímulos circularmente simétricos modulados pela função cilíndrica de Bessel de ordem zero $\left(\mathrm{J}_{0}\right)$. Essa classe de estímulo elementar tem sido denominada frequiência radial, porque a modulação de contraste varia de acordo com o seno ou co-seno na direção radial, considerando o centro do círculo como a origem do sistema de coordenadas polares (Figura 1). Kelly partiu da idéia de que padrões radiais circularmente simétricos definidos em coordenadas polares poderiam ser mais apropriados para estudar o SVH, considerando a simetria aproximadamente circular da retina (Kelly, 1960), e mais naturais, por fornecerem um centro claro de fixação (Kelly \& Magnuski, 1975). O mesmo não acontece com padrões definidos em coordenadas cartesianas, por exemplo, grade senoidal (Figura 1). Grade senoidal é um estímulo elementar cuja luminância varia de acordo com o seno ou co-seno, em uma direção no espaço, em um sistema de coordenadas cartesianas (Figura 1).

Em 1975 Kelly e Magnuski mensuraram a FSC para frequiências radiais em adultos. O principal objetivo de Kelly e Magnuski foi comparar a resposta de adultos para estímulos radiais e grades senoidais. Em 1982 Kelly replicou parte do estudo de 1975 com a técnica psicofísica de ajustamento de contraste, no qual o participante ajustava seu próprio limiar de contraste. Os resultados obtidos por Kelly nos dois estudos com adultos demonstraram que a faixa de maior sensibilidade para estímulos radiais foi por volta de $0,5-2,0 \mathrm{cpg}$. Os resultados de
Kelly também mostraram que o SVH era mais sensível para grades senoidais do que para estímulos radiais.

Simas, Santos e Thiers (1997) mensuraram e compararam a sensibilidade ao contraste de adultos para padrões definidos matematicamente em coordenadas cartesianas (p.ex., grades senoidais) e padrões definidos em coordenadas polares ( $\mathrm{p}$. ex., estímulos radiais e angulares). Os resultados de Simas e colaboradores (1997) foram semelhantes aos resultados de Kelly (Kelly, 1982; Kelly \& Magnuski, 1975). Isso é, o sistema visual de adultos foi mais sensível para grades senoidais do que para estímulos radiais modulados pela função esférica de Bessel. Outros resultados e informações relacionadas à definição matemática e variação física de contraste em estímulos radiais podem ser encontrados em Simas e Santos (2002b).

\section{Envelhecimento humano e a FSC}

Mudanças significativas nas características neurais e ópticas do SVH com a idade originaram vários estudos tentando relacionar o envelhecimento à FSC de grade senoidal vertical (Arden \& Jacobsen, 1978; Arundale, 1978; Elliott, Whitaker \& MacVeigh, 1990; Owsley, Sekuler \& Siemsen, 1983; Schefrin, Tregear, Harvey Jr \& Werner, 1999; Sekuler, Hutman \& Owsley, 1980). Embora não exista um consenso sobre as bandas de freqüências espaciais que são afetadas pelo envelhecimento, estudos psicofísicos em humanos com grade senoidal vertical relatam reduções gradativas com a idade, principalmente nos mecanismos que processam bandas de freqüências espaciais médias e altas (Elliott \& cols., 1990; Fiorentini, Porciatti, Morrone \& Burr, 1996; Schefrin \& cols., 1999). Estudos relatam também declínio significativo na FSC apenas depois dos 50 anos (Higgins, Jaffe, Caruso \& deMonasterio, 1988; Nio \& cols., 2000; Owsley \& cols., 1983).

\section{O presente estudo}

A maior parte das pesquisas relacionando percepção visual de contraste (p.ex., FSC) e o processo de envelhecimento foi realizada com padrões espaciais do tipo grade senoidal (Nio \& cols., 2000; Owsley \& cols.,1983; Schefrin \& cols., 1999). Não encontramos nenhum estudo utilizando padrões espaciais radiais ou estímulos visuais circularmente simétricos para mensurar a FSC de idosos. Nesse sentido, o presente estudo procurou caracterizar respostas típicas do sistema visual para padrões radiais circularmente simétricos em adultos e idosos. Caracterizou, ainda, a resposta dos mesmos participantes, nas mesmas condições, para grade senoidal, para efeito de comparação. O objetivo principal foi descrever os efeitos do envelhecimento na detecção de estímulos radiais utilizando o método psicofísico da escolha forçada. Pesquisa dessa natureza parte da proposição de que estímulos em coordenadas cartesianas ( $p$. ex., grade senoidal) e estímulos em coordenadas polares (p. ex., frequiências radiais) são processados por vias ou áreas visuais distintas conforme descritos por estudos psicofísicos (Santos \& cols., 2004; Santos, Nogueira \& Simas, 2005; Santos \& Simas, 2002; Santos, Simas \& Nogueira, 2003; Wilson \& Wilkinson, 1998; Wilson, Wilkinson \& Asaad, 1997) e neurofisiológicos (Gallant, Brau \& van Essen, 1993; Gallant, Connor, Rakshit, Lewis \& van Essen, 1996; Heywood, Gadotti \& Cowey, 1992; Merigan, 1996; Wilkinson \& cols., 2000). 


\section{Método}

\section{Participantes}

Participaram dos experimentos 12 voluntários, seis adultos jovens com idade entre $19-23$ anos $(M=21,4 ; D P=1,23)$ e seis idosos com idade entre $60-65$ anos $(M=63,6 ; D P=1,67)$. Todos os participantes apresentavam acuidade visual normal ou corrigida e tinham consultado os seus oftalmologistas havia menos de 12 meses. Os mesmos também se encontravam livres de doenças oculares identificáveis. A participação na pesquisa ocorreu mediante a assinatura de termo de consentimento livre e esclarecido e conforme a Resolução no 196/96 do Conselho Nacional de Saúde, que trata das diretrizes e normas de pesquisas envolvendo seres humanos.

\section{Equipamentos e Estímulos}

Os estímulos radiais e grades senoidais foram gerados em um monitor de vídeo CLINTON MEDICAL monocromático digital de 21 polegadas, de alta resolução (1280x1024), controlado por um microcomputador. Uma cadeira foi fixada a $150 \mathrm{~cm}$ da tela do monitor de vídeo. A luminância média da tela foi de $0,7 \mathrm{~cd} / \mathrm{m}^{2}$ ajustada por um fotômetro do tipo SPOT METTER, com precisão de um grau, ASAHI PENTAX. A luminância mínima da tela foi de $0,5 \mathrm{~cd} / \mathrm{m}^{2}$ e a máxima de $0,9 \mathrm{~cd} / \mathrm{m}^{2}$.

As frequiências acromáticas utilizadas para mensurar a sensibilidade ao contraste para grades senoidais (FSC) e padrões radiais (FSCr) foram $0,25,0,5,1,0$ e 2,0 ciclos por grau de ângulo visual (cpg). Estas eram circulares, com um diâmetro de 7 graus de ângulo visual a $150 \mathrm{~cm}$ de distância da tela, e foram apresentadas em tempo real no monitor (Figura 1).

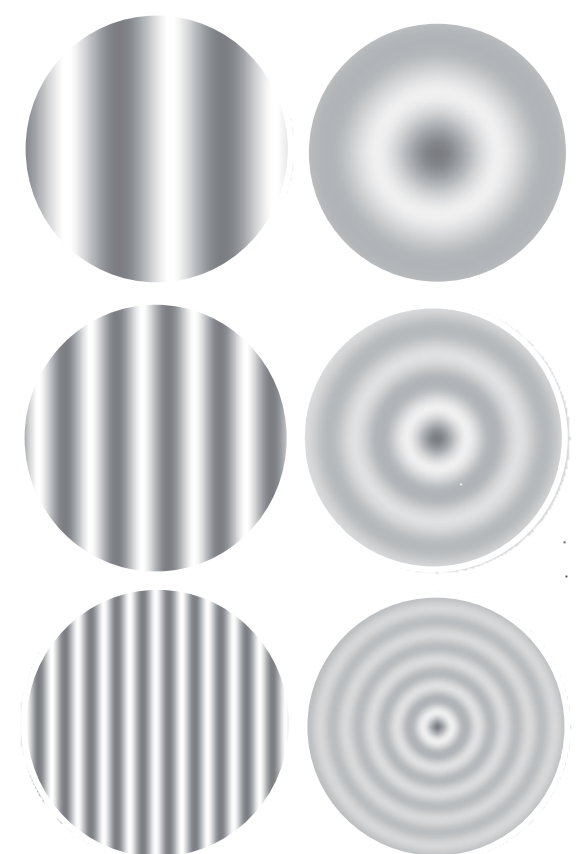

Figura 1. Exemplos de estímulos de freqüências espaciais à esquerda e frequiências radiais à direita, de cima para baixo, 0,5, 1,0 e 2,0 cpg, respectivamente. Estímulos originalmente calibrados para serem vistos a $150 \mathrm{~cm}$ de distância.

\section{Procedimento}

As estimativas foram realizadas com método psicofísico da escolha forçada (Santos \& cols., 2003, 2004, 2005; Santos \& Simas, 2002; Simas \& Santos, 2002a, 2002b; Wetherill \& Levitt, 1965). Esse método baseia-se no cálculo da probabilidade de acertos consecutivos por parte do participante, ou seja, em cerca de 120 apresentações de escolhas entre os dois estímulos, o estímulo de teste (grade senoidal ou freqüência radial) é percebido $79 \%$ das vezes pelo participante. O procedimento para medir o limiar de contraste para cada padrão consistiu na apresentação sucessiva simples do par de estímulos e o participante teria que escolher qual entre eles continha a grade senoidal ou freqüência radial. O estímulo neutro foi sempre um padrão homogêneo com luminância média de $0,7 \mathrm{~cd} / \mathrm{m}^{2}$. O critério adotado para variar o contraste da freqüência radial ou da grade senoidal (estímulo de teste) era o de três acertos consecutivos para decrescer uma unidade e um erro para acrescer da mesma unidade $(0,08 \%)$.

Durante cada sessão experimental era apresentada uma sequiência de estímulos que foi iniciada com um sinal sonoro seguido imediatamente pela apresentação do primeiro estímulo por $2 \mathrm{~s}$, seguido de um intervalo entre estímulos de $1 \mathrm{~s}$, seguido pela apresentação do segundo estímulo por 2 s e da resposta do participante. A ordem de apresentação dos estímulos era aleatória. Se a resposta fosse correta, era seguida por outro sinal sonoro e um intervalo de $3 \mathrm{~s}$ para a sequiência se repetir. O sinal sonoro que indicava o início da apresentação do par de estímulos e o que indicava a escolha correta eram diferentes. A sessão experimental variava em duração dependendo dos erros e acertos do participante até proporcionar um total de 10 reversões (cinco máximos e cinco mínimos), conforme requerido para o final automático da mesma.

Cada uma das freqüências utilizadas para mensurar a FSC e FSCr foi estimada pelo menos duas vezes (duas sessões experimentais), em dias diferentes, por cada um dos participantes. No total, 48 curvas foram medidas, sendo 24 curvas para cada grupo de participantes (12 para grade senoidal e 12 para padrões radiais). Todas as estimativas foram medidas à distância de $150 \mathrm{~cm}$, com visão binocular.

Os participantes foram sentados individualmente em uma cadeira fixada a $150 \mathrm{~cm}$ da tela do monitor e foram orientados a pressionar o botão do lado esquerdo do mouse quando o estímulo de teste (grade senoidal ou freqüência radial) fosse apresentado primeiro, e o botão do lado direito quando fosse apresentado em segundo lugar, isso é, após o estímulo cinza (ou neutro).

Todos os participantes receberam a seguinte instrução: "será apresentado uma seqüência de pares de estímulos, um com listras claras e escuras (ou círculos claros e escuros) e outro totalmente cinza. Você deverá escolher sempre o estímulo que contém as listras (ou os círculos), pressionando o botão do lado esquerdo do mouse quando o estímulo com listras (ou círculos) for apresentado primeiro e, o botão do lado direito, quando for apresentado em segundo lugar (após o estímulo cinza). Sempre que a sua escolha for correta, a mesma será imediatamente seguida por um sinal sonoro". Os experimentos só começaram quando o experimentador certificou-se que os participantes entenderam e responderam conforme as instruções. 


\section{Resultados}

Os valores de contrastes máximos e mínimos obtidos para cada ponto (ou frequiência) foram agrupados em planilhas por estímulos (grade senoidal ou radial) e por grupo (adultos ou idosos), e a grande média foi utilizada como estimativa da sensibilidade ao contraste, que é o inverso do limiar de contraste.

A Figura 2A mostra a função de sensibilidade ao contraste de adultos e idosos para freqüências espaciais, grades senoidais (FSC). A Figura 2B mostra a função de sensibilidade ao contraste de adultos e idosos para frequiências radiais (FSCr). A Figura 2C mostra a FSC e FSCr dos adultos e a Figura 2D mostra a FSC e FSCr dos idosos. As frequiências espaciais e radiais são apresentadas nos gráficos em função da sensibilidade ao contraste do SVH. As barras verticais indicam os erros padrões das médias com intervalos de $99 \%$ de confiança corrigidos para o tamanho da amostra pelo estatístico $t$-Student.

Observa-se na Figura 2A que a faixa de maior sensibilidade para freqüências espaciais (FSC) ficou em 0,5 cpg para os adultos e idosos, porém, os adultos foram da ordem 1,4 vezes mais sensíveis do que os idosos na faixa de máxima sensibilidade ao contraste. Já a Figura 2B mostra que a faixa de máxima sensibilidade para freqüências radiais (FSCr) ficou em $0,25 \mathrm{cpg}$ para os dois grupos, entretanto os adultos foram da ordem de 2,2 vezes mais sensíveis do que os idosos na faixa de máxima sensibilidade. Esses resultados demonstram que as alterações relacionadas à idade na faixa de máxima sensibilidade foram maiores para estímulos radiais (Figura 2B) do que para freqüências espaciais (Figura 2A).

Por outro lado, a Figura $2 \mathrm{C}$ demonstra que a sensibilidade ao contraste dos adultos foi da ordem de 1,4, 2,5, 2,7 e 1,4 vezes maior para frequiências espaciais (FSC) do que para freqüências radiais ( $\mathrm{FSCr}$ ) nas freqüências de 0,25, 0,5, 1,0 e 2,0 cpg, respectivamente. A Figura 2D mostra que a sensibilidade ao contraste dos idosos foi da ordem de 3,4 , 4,0, 2,5 e 1,1 vezes maior para frequiências espaciais (FSC) do que para frequiências radiais (FSCr) nas freqüências de $0,25,0,5,1,0$ e 2,0 cpg, respectivamente. Em outras palavras, esses resultados mostraram que as diferenças entre as curvas de FSC e FSCr dos adultos foram maiores nas frequiências intermediárias (p. ex., 0,5 e 1,0 cpg; Figura 2C), enquanto as diferenças entre as curvas de FSC e FSCr dos idosos foram maiores nas frequiências mais baixas (p. ex., 0,25 e 5,0 cpg; Figura 2D).

A ANOVA para medidas repetidas mostrou diferenças significativas entre a FSC de adultos e idosos para grades senoidais $\left[F_{1,684}=110,6 ; p<0,001\right]$ e para frequiências radiais $\left[F_{1,684}=110,6 ; p<0,001\right]$. Mostrou ainda diferenças significativas entre a FSC e a FSCr de adultos $\left[F_{1,684}=310,3 ; p<0,001\right]$ e entre a FSC e a FSCr de idosos $\left[F_{1684}=215,4 ; p<0,001\right]$. As análises usando o teste post-hoc Tukey HSD mostraram diferenças estatisticamente significantes entre o grupo de adultos e idosos em todas as frequiências espaciais (i.e., $p<0,023$ para $0,25 \mathrm{cpg} ; p<0,001$ para $0,5 \mathrm{cpg} ; p<0,001$ para $1,0 \mathrm{cpg}$ e $p<0,001$ para $2,0 \mathrm{cpg}$ ) e em todas as frequiências radiais (i.e., $p<0,001$ para $0,25 \mathrm{cpg} ; p<0,001$ para $0,5 \mathrm{cpg}$;
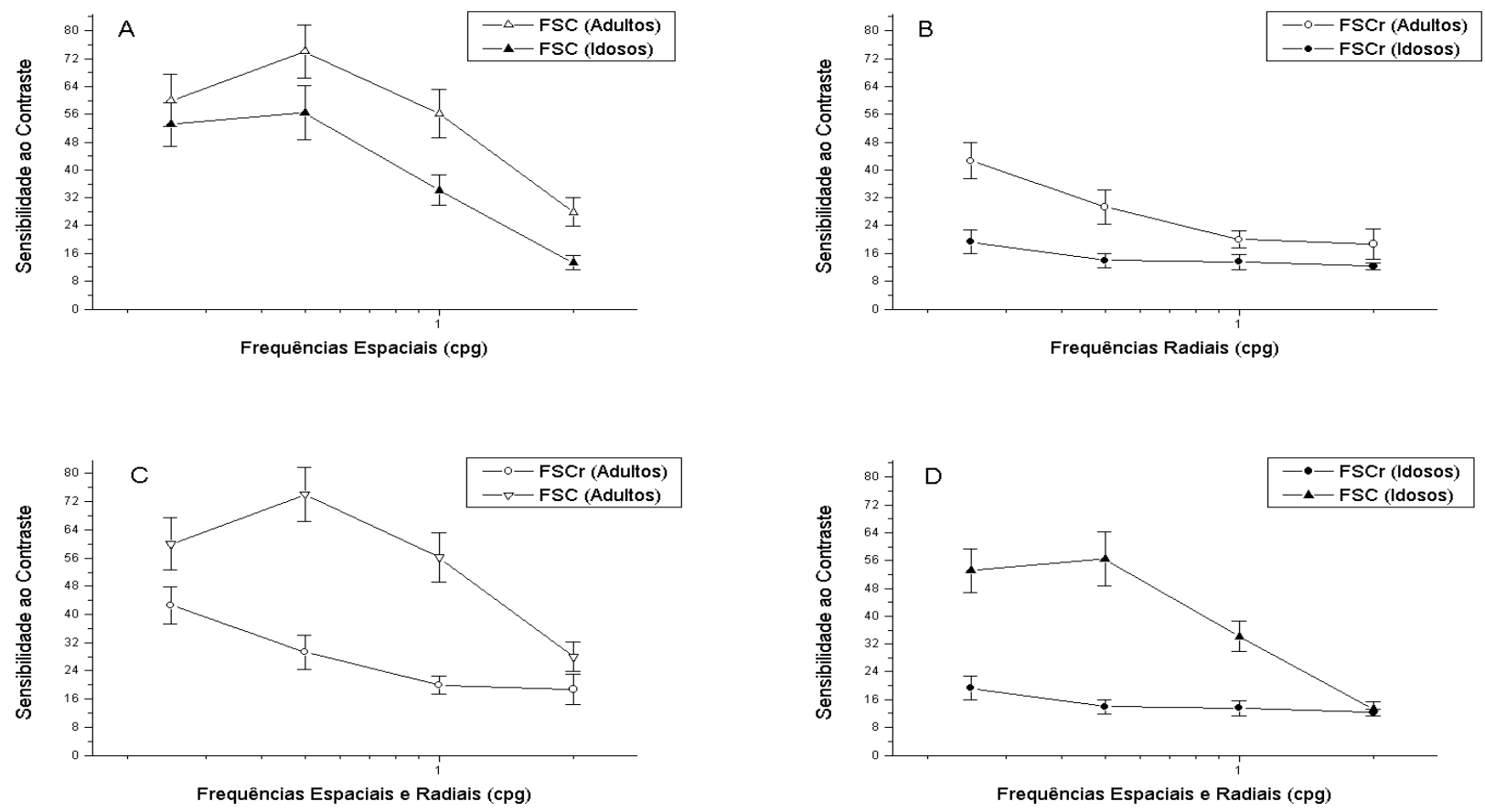

Figura 2. Estimativas da sensibilidade ao contraste em função de freqüências espaciais (FSC) e de freqüências radiais (FSCr). (A) Curvas de adultos e idosos para frequiências espaciais. (B) Curvas de adultos e idosos para freqüências radiais. (C) Curvas de adultos para frequiências espaciais e radiais. (D) Curvas de idosos para frequiências espaciais e radiais. As barras verticais indicam os erros padrões das médias com intervalos de $99 \%$ de confiança corrigidos para o tamanho da amostra pelo estatístico $t$-Student. 
$p<0,001$ para 1,0 cpg e $p<0,001$ para 2,0 cpg). Assim, essas análises, utilizando nível de significância de $5 \%(\alpha=0,05)$, mostraram diferenças significantes entre adultos e idosos em todas as frequiências espaciais e radiais. Mostraram ainda que a menor diferença entre adultos e idosos ocorreu na frequiência espacial de 0,25 cpg ( $p<0,023$; Figura 2A).

Em linhas gerais, os resultados demonstraram que a sensibilidade ao contraste ou a detecção de estímulos de freqüências espaciais (FSC) e de frequiências radiais (FSCr) declina com o envelhecimento humano. Encontramos ainda que o sistema visual de adultos e de idosos é mais sensível para estímulos do tipo grade senoidal (ou freqüência espacial) do que para estímulos radiais circularmente simétricos. Esses resultados são consistentes com a existência de mecanismos distintos sensíveis a estímulos circularmente simétricos em coordenadas polares.

\section{Discussão}

Este estudo focaliza pelo menos duas questões básicas relacionadas ao processamento visual da forma e o envelhecimento do sistema visual. Primeiro, utiliza a sensibilidade ao contraste para descrever alterações na performance do SVH relacionadas à idade para estímulos radiais e grades senoidais, especificando as bandas ou faixas de frequiências mais afetadas. Segundo, caracteriza a resposta para cada conjunto de estímulos e investiga para qual deles o sistema visual apresenta maior sensibilidade ou detecta melhor. Questões como essas demonstram a necessidade de desenvolver pesquisas para acompanhar e estudar mecanismos sensoriais básicos durante o processo de envelhecimento, procurando descrever e aperfeiçoar o instrumento psicofísico capaz de caracterizar e acompanhar respostas do SVH com e sem patologias.

\section{Sensibilidade ao contraste de adultos versus idosos}

Os nossos resultados mostram que a sensibilidade ao contraste dos adultos para grades senoidais (FSC, Figura 2A) e para freqüências radiais (FSCr, Figura 2B) é melhor do que a dos idosos. Isso é, ocorreram reduções significativas na FSC e na FSCr dos idosos comparados a FSC e FSCr dos adultos (Figuras 2A e 2B). Alterações na FSC relacionadas ao processo de envelhecimento já eram, de certa forma, esperadas, considerando os principais achados da literatura para freqüências espaciais (ou grade senoidal) em coordenadas cartesianas (Benedek \& cols., 2003; Kackson, Owsley, Cordle \& Finley, 1998; Owsley \& cols., 1983). Por outro lado, embora a redução na $\mathrm{FSCr}$ relacionada ao processo de envelhecimento fosse esperada, baseado nos dados com grades senoidais verticais e nos trabalhos com estímulos de freqüências angulares definidos em coordenadas polares (Santos \& cols., 2003, 2004, 2005), não esperávamos encontrar alterações significativas relacionadas à idade para todas as freqüências radiais, especialmente nas freqüências radiais mais baixas, 0,25 e $0,5 \mathrm{cpg}$, pois as pesquisas citadas anteriormente com grade senoidal e estímulos angulares relataram redução apenas nas frequiências médias e altas. No presente estudo, as diferenças entre adultos e idosos foram da ordem de 1,3, 1,4, 1,8 e 2 nas frequiências espaciais de 0,25, 0,5, 1,0 e 2,0 cpg, respectivamente (Figura 2A), e da ordem de 2,2, 2,1, 1,5 e 1,4 para as freqüências radiais de 0,25, 0,5, 1,0 e 2,0 cpg, respectivamente (Figura 2B). Assim, a FSCr dos idosos comparada a dos adultos mostrou maior prejuízo nas frequiências radiais mais baixas (i.e., 0,25 e 0,5 cpg) enquanto a FSC dos idosos mostrou maior prejuízo nas frequiências espaciais mais altas (i.e, 1 e $2 \mathrm{cpg}$ ).

Entretanto, é cedo para afirmar que o processo de envelhecimento atue diferentemente sobre os mecanismos responsáveis pelo processamento de frequiências espaciais e frequiências radiais, embora alguns estudos sugiram que o envelhecimento altere as vias visuais discretamente (Elliott \& cols., 1990; Habak \& Faubert, 2000; Schefrin \& cols., 1999; Sekuler \& cols., 1980). Contudo, os nossos resultados com idosos e frequiências radiais são sugestivos e novos experimentos com luminâncias mais altas e faixas etárias diferentes precisam ser conduzidos para investigar e aprofundar os possíveis efeitos do envelhecimento nos mecanismos responsáveis pelo processamento de contraste em padrões radiais circularmente simétricos.

\section{Sensibilidade de adultos e idosos para estímulos radiais versus grades senoidais}

O fato de o sistema visual dos adultos ser mais sensível para grades senoidais do que para estímulos radiais (Figura 2C) já era esperado, pois Kelly e Simas já haviam demonstrado (Kelly, 1982; Kelly \& Magnuski, 1975; Simas \& Santos, 2002b; Simas \& cols., 1997). Entretanto, este estudo mostrou que o sistema visual dos idosos também é mais sensível para grades senoidais, FSC, do que para frequiências radiais, $\mathrm{FSCr}$ (Figura 2D). Essa redução na FSCr, comparada a FSC, pode estar relacionada à própria definição matemática dos estímulos radiais que variam de acordo com a função matemática de Bessel. Os estímulos radiais apresentam contraste mais intenso no centro e menos intenso na periferia (Figura 1, ver também Simas \& Santos, 2002b) tendendo a ser constante no infinito. Neste contexto, uma hipótese para a redução na FSCr comparada à FSC pode ser devido aos estímulos radiais não possuírem amplitude equivalente ao longo do estímulo, isso é, eles apresentam amplitude máxima no centro (fóvea), diminuindo para periferia do campo visual (Simas \& Santos, 2002b). Ao contrário, a amplitude da grade senoidal é equivalente ao longo de todo o estímulo ou campo visual (Figura 1). Então, é possível que a quantidade de canais ou a forma (intensidade) com que os canais específicos para cada padrão sejam ativados (na fóvea e próximo à fóvea) façam a diferença. A relação lógica por trás desse processo é que, se os dois padrões ativam canais específicos de formas diferentes, aquele que ativa mais intensamente o maior número de canais poderia apresentar maior sensibilidade.

Inclusive, ao observar a sensibilidade ao contraste de adultos e idosos para estímulos radiais e grade senoidal (Figuras 2A, 2B, 2C e 2D), parece que cada conjunto de estímulo tem atributos peculiares relacionados à detecção da forma e do contraste que os diferenciam entre si. As alterações na sensibilidade do SVH para esses estímulos são difíceis de serem explicadas por um mecanismo único. Essa hipótese pode ser reforçada por estudos psicofísicos (Santos \& Simas, 2002; Simas \& Santos, 2002a, 2002b; Simas \& cols., 1997; Wilson \& Wilkinson, 1998; Wilson \& cols., 1997) e 
neurofisiológicos (Gallant \& cols., 1996; Heywood \& cols., 1992; Merigan, 1996; Wilkinson \& cols., 2000), que sugerem que grade senoidal e padrão radial são processados por mecanismos e áreas visuais distintas. Por exemplo, em 1994, Kobatake e Tanaka encontraram células seletivas a estímulos radiais na área visual V2, enquanto Gallant e colaboradores (1993, 1996) encontraram evidências para estímulos radiais na área visual V4. Nesse contexto, é possível que o processo de envelhecimento altere naturalmente tanto a área visual V1, envolvida no processamento de grade senoidal, como as áreas visuais V4 e IT (córtex ínfero-temporal), envolvidas no processamento de estímulos em coordenadas polares.

\section{Considerações Finais}

Esses resultados demonstram que a capacidade que o SVH possui para detectar estímulos depende da idade (envelhecimento) e das características físicas e matemáticas que definem a modulação do contraste. Essas características, entre outras, por sua vez, podem estar relacionadas à detecção, ao processamento neural e à integração final da imagem percebida. Levando em conta essas considerações, os nossos resultados e os dados encontrados na literatura, podemos inferir que o sistema visual utiliza mecanismos ou vias distintas para processar grades senoidais e padrões radiais e que o envelhecimento, possivelmente, afeta os dois sistemas.

\section{Referências}

Akutsu, H. \& Legge, G. E. (1995). Discrimination of compound gratings: Spatial-frequency channels or local features? Vision Research, 35, 2685-2695.

Arden, G. B. \& Jacobsen, J. (1978). A simple grating test for contrast sensitivity: Preliminary results indicate value for screening in glaucoma. Investigative Ophthalmology \& Visual Science, 17, 23-32.

Arundale, K. (1978). An investigation into the variation of human contrast sensitivity with age and ocular pathology. British Journal of Ophthalmology, 62, 213-215.

Benedek, G., Benedek, K., Kéri, S. \& Janáky, M. (2003). The scotopic low-frequency spatial contrast sensitivity develops in children between the ages of 5 and 14 years. Neuroscience Letters, 345, 161-164.

Blakemore, C. \& Campbell, F. C. (1969a). Adaptation to spatial stimuli. Journal of Physiology, 200, 11-12.

Blakemore, C. \& Campbell, F. C. (1969b). On the existence of neurons in the human visual system selectively sensitive to the orientation and size of retinal images. Journal of Physiology, 203, 237-260.

Bour, L. J. \& Apkarian, P. (1996). Selective broad-band spatial frequency loss in contrast sensitivity functions. Investigative Ophthalmology \& Visual Science, 37(12), 2475-2484.

Braddick, O., Campbell, F. W. \& Atkinson, J. (1978). Channels in vision: Basic aspects. Em R. Held, H. W. Leibowitz \& H. L. Teuber (Orgs.), Handbook of Sensory Physiology: Perception. (Vol. V, pp. 3-38). New York: Springer-Verlag.

Campbell, F. W. \& Robson, F. G. (1968). Application of the Fourier analysis to the visibility of gratings. Journal of Physiology, 197, 551-566.
Candy, T. R., Crowell, J. A. \& Banks, M. S. (1998). Optical, receptoral, and retinal constraints on foveal and peripheral vision in the human neonate. Vision Research, 38, 38573870.

Cornsweet, T. N. (1970). Vision perception. New York: Academic Press.

Dakin, S. C. \& Hess, R. F. (1997). The spatial mechanisms mediating symmetry perception. Vision Research, 37(20), 2915-2939.

De Valois, R. L. \& De Valois, K. K. (1988). Spatial Vision. New York: Oxford University Press.

Elliott, D. B. \& Situ, P. (1998). Visual acuity versus letter contrast sensitivity in early cataract. Vision Research, 38, 2047-2052.

Elliott, D, Whitaker, D. \& MacVeigh, D. (1990). Neural contribution to spatiotemporal contrast sensitivity decline in healthy ageing eyes. Vision Research, 30, 541-547.

Fiorentini, A., Porciatti, V., Morrone, M. C. \& Burr, D. C. (1996). Visual ageing: Unspecific decline of the responses to luminance and colour. Vision Research, 3, 3557-3566.

Gallant, J. L., Brau, J. \& van Essen, D. C. (1993). Selectivity for polar hyperbolic, and cartesian gratings in macaque visual cortex. Science, 259, 100-103.

Gallant, J. L., Connor, C. E., Rakshit, S., Lewis, J. W. \& van Essen, D. C. (1996). Neural responses to polar, hyperbolic, and cartesian gratings in area V4 of the macaque monkey. Journal of Neurophysiology, 76, 2718-2739.

Georgeson, M. A. \& Sullivan, G. D. (1975). Contrast Constancy: Deblurring in human vision by spatial frequency channels. Journal of Physiology, 252, 627-656.

Graham, N. (1972). Spatial frequency channels in the human visual system: Effects of luminance and pattern drift rate. Vision research, 12, 53-63.

Graham, N. \& Nachmias, J. (1971). Detection of grating patterns containing two spatial frequencies: A comparison of singlechannel and multiple channel models. Vision Research, 11, 251-259.

Habak, C. \& Faubert, J. (2000). Larger effect of aging on the perception of higher-order stimuli. Vision Research, 40, 943-950.

Heywood, C. A., Gadotti, A. \& Cowey, A. (1992). Cortical area V4 and its role in the perception of color. Journal of Neuroscience, 12, 4056-4065.

Higgins, K. E, Jaffe, M. J., Caruso, R. C. \& deMonasterio, F. M. (1988). Spatial contrast sensitivity: effects of age, test-retest, and psychophysical method. Journal of the Optical Society of America A, 5, 2173-2180.

John, R. S. (1997). Contrast detection and orientation discrimination thresholds associated with meridional amblyopia. Vision Research, 37, 1451-1457.

Kackson, G. R., Owsley, C. Cordle, E. P. \& Finley, D. F. (1998). Aging and scotopic sensitivity. Vision Research, 38, 3655-3662.

Kelly, D. H. (1960). J stimulus patterns for vision research. Journal of the Optical Society of America, 50, 1115-1116.

Kelly, D. H. (1982). Motion and vision: IV. Isotropic and anisotropic spatial response. Journal of the Optical Society of America, 72, 432-439.

Kelly, D. H. \& Magnuski, H. S. (1975). Pattern detection and the two dimensional Fourier transform: Circular targets. Vision Research, 15, 911-915.

Kobatake, E. \& Tanaka, K. (1994). Neuronal selectivities to complex object features in the ventral visual pathway of the macaque cerebral cortex. Journal of Neurophysiology, 71(3), 856-867. 
Menees, S. M. (1998). The effect of spatial frequency adaptation on the latency of spatial contrast detection. Vision Research, 38, 3933-3942.

Merigan, W. H. (1996). Basic visual capabilities and shape discrimination after lesions of extrastriate area $\mathrm{V} 4$ in macaques. Visual Neuroscience, 13, 51-60.

Nio, Y. K., Jansonius, N. M., Fidler, V., Geraghty, E., Norrby, S. \& Kooijman, A. C. (2000). Age-related changes of defocusspecific contrast sensitivity in healthy subjects. Ophthalmic and Physiological Optics, 20, 323-334.

O’ Donnell, B. F., Nestor, P. G., Potts, G. F., Stylianopoulos, K. C., Shenton, M. E. \& McCarley, R. W. (2002). Spatial frequency discrimination in schizophrenia. Journal of Abnormal Psychology, 111(4), 620-625.

Olds, E. S. \& Engel, S. A. (1998). Linearity across spatial frequency in object recognition. Vision Research, 38, 2109-2118.

Owsley, C., Sekuler, R. \& Siemsen, D. (1983). Contrast sensitivity throughout adulthood. Vision Research, 23, 689-699.

Sachs, M. B., Nachmias, J. \& Robson, J. G. (1971). Spatial frequency channels in human vision. Journal of the Optical Society of America, 61, 1176-1186.

Santos, N. A. \& Simas, M. L. B. (2001). Percepção e processamento visual da forma: Discutindo modelos teóricos atuais. Psicologia: Reflexão \& Crítica, 14(1), 151-160.

Santos, N.A. \& Simas, M. L. B. (2002). Percepção e processamento visual da forma em humanos: Filtros de freqüências radiais de 1 e 4 cpg. Psicologia: Reflexão e Crítica, 15(2), 383-391.

Santos, N. A., Simas, M. L. B. \& Nogueira, R. M. T. B. L. (2003). Processamento visual da forma em idosos: Curvas de limiar de contraste para freqüências angulares e senoidais. Psicologia: Reflexão e Crítica, 16(2), 271-277.

Santos, N. A., Simas, M. L. B. \& Nogueira, R. M. T. B. L. (2004). Comparison of angular frequency contrast sensitivity in Young and older adults. Brazilian Journal of Medical and Biological Research, 37, 375-378.

Santos, N. A., Nogueira, R. M. T. L. \& Simas, M. L. B. (2005). Processamento visual da forma: Evidências para canais múltiplos de frequiências angulares em humanos. Psicologia Reflexão e Crítica, 18(1), 98-103.

Schefrin, B. E., Tregear, S. J., Harvey Jr, L. O. \& Werner, J. S. (1999). Senescent changes in scotopic contrast sensitivity. Vision Research, 39, 3728-3736.

Sekuler, R., Hutman, L. P. \& Owsley, C. (1980). Human aging and spatial vision. Science, 209, 1255-1256.

Simas, M. L. S. \& Santos, N. A. (2002a). Narrow-band 1, 2, 3, 4, 8,16 and 24 cycles $/ 360^{\circ}$ angular frequency filters. Brazilian Journal of Medical and Biological Research, 35, 243-253.
Simas, M. L. S. \& Santos, N. A. (2002b). Contrast sensitivity to radial frequencies modulated by Jn and jn Bessel profiles. Brazilian Journal of Medical and Biological Research, 35, 1357-1366.

Simas, M. L. S., Santos, N. A. \& Thiers, F. A. (1997) Contrast sensitivity to angular frequency stimuli is higher than that for sine wave gratings in the respective middle range. Brazilian Journal of Medical and Biological Research, 30, 633-636.

Slaghuis, W. L. (1998). Contrast sensitivity for stationary and drifting spatial frequency gratings in positive- and negativesymptom schizophrenia. Journal of Abnormal Psychology, 107(1), 49-62.

Van Sluyters, R. C., Atkinson, M. S., Held, R. M., Hoffman, K. \& Shatz, C. J. (1990). The development of vision and visual perception. Em S. W. Spillmann \& J. S. Werner (Orgs.), The Neurophysiological Foundations (pp. 349-379). New York: Academic Press.

Wetherill, G. B. \& Levitt, H. (1965). Sequential estimation of points on a psychometric function. The British Journal of Mathematical and Statistical Psychology, 48, 1-10.

Wilkinson, F., James, T. W., Wilson, H. R., Gati, J. S., Menon, E. S. \& Goodale, M. A. (2000). An fMRI study of the selective activation of human extrastriate form vision areas by radial and concentric gratings. Current Biology, 10, 1455-1458.

Wilson, H. R., Levi, D., Maffei, L., Rovamo, J. \& De Valois, R. (1990). The perception of form: Retina to striate cortex. Em S. W. Spillmann \& J. S. Werner (Orgs.), Visual Perception: The Neurophysiological Foundation (pp. 231-271). New York: Academic Press.

Wilson, H. R. \& Wilkinson, F. (1998). Detection of global structure in glass patterns: Implications for form vision. Vision Research, 38, 2933-2947.

Wilson, R. W., Wilkinson, F. \& Asaad, W. (1997). Concentric orientation summation in human form vision. Vision Research, $37,2325-2330$.
Recebido em 13.01.2006

Primeira decisão editorial em 02.05.2006

Versão final em 25.09.2006

Aceito em 28.03.2007 